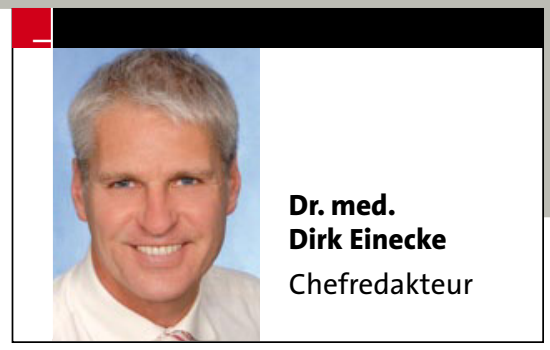

In den meisten Gesundheitssystemen werden Ärzte nach der Quantität ihrer Leistungen honoriert. In Großbritannien stellte man 2004 auf Bezahlung nach Qualität um (Pay-for-Performance). Der Erfolg der Maßnahme wurde jetzt anhand der Daten von ca. 470000 Hypertonikern ausgewertet, deren Hochdruck zwischen 2000 und 2007 diagnostiziert worden war. Die Inzidenz an Herzinfark- ten, Schlaganfällen, Nierenversagen und die Gesamtmortalität unterschied sich bei den Patienten, deren Ärzte nach dem Prinzip des Pay-for-Performance honoriert worden waren, nicht von denen der Vergleichsgruppe. Die Qualität ärztlicher Leistung lässt sich nicht einfach durch finanzielle Anreize steigern, so die Autoren der Studie.

BMJ, 2011; 342 (jan25 3): d108 DOI: 10.1136/bmj.d108

WENN DIE CHEMIE NICHT STIMMT

\title{
Schwierige Patienten: nach Arztbesuch kränker
}

Ein schlechtes Arzt-Patienten-Verhältnis wirkt sich negativ auf den Erfolg der ärztlichen Bemühungen aus: 750 Patienten, die eine Klinikambulanz aufsuchten, wurden vor der Konsultation nach ihren Erwartungen und ihrem Gesundheitszustand befragt. Nach zwei Wochen sollten sie mitteilen, ob sich ihre Symptome verbessert hätten und wie sie den Arzt beurteilten. Die Ärzte wiederum wurden befragt, ob sie den Patienten als besonders schwierig empfunden hätten. Bei „schwierigen“ Patienten und bei solchen, die angaben, ihren Arzt als nicht vertrauenswürdig empfunden zu haben, hatten sich die Symptome signifikant häufiger verschlimmert.

Journal of General Internal Medicine, 2011; DOI: 10.1007/s11606-010-1620-6

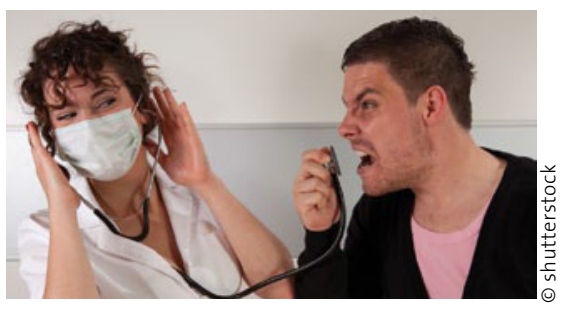

Schlechter Start in die Therapie.

\section{BEGINN EINER HOCHDRUCKTHERAPIE}

\section{Besser nicht mit HCTZ}

Das Diuretikum Hydrochlorothiazid (HCTZ) senkt den Blutdruck schlechter als ACEHemmer, $\mathrm{AT}_{1}$-Antagonisten, Betablocker oder Kalziumantagonisten. Dies ergab eine Metaanalyse von 14 Studien, in denen die Wirksamkeit der verschiedenen Substanzklassen mittels 24-Stunden-Blutdruckmessung verglichen wurde. Mit HCTZ (12,5 und $25 \mathrm{mg} / \mathrm{d}$ ) wurde gerade mal eine RR-Senkung von 6,5/4,5 mmHg erreicht. Vor allem nachts und in den frühenO Morgenstunden schwächelte HCTZ. Die 50-mg-Dosis ist zwar stärker wirksam, aber mit mehr Nebenwirkungen (Hypokaliämie, Insulinresistenz) belastet. Zudem gibt es kaum Studien zum Nutzen hinsichtlich Morbidität und Mortalität. Prof. F. Messerli, New York, empfiehlt daher, HCTZ bei Hypertonie nicht als erstes Medikament zu verordnen. Als Kombinationspartner ist es jedoch wertvoll: Kombiniert mit einem Hemmstoff des Renin-Angiotensin-Systems verstärkt HCTZ dessen antihypertensive Wirkung.

J Am Coll Cardiol 2011;57:590-600

\section{MENOPAUSE}

\section{Schützen Wallungen vor Brustkrebs?}

Auch unangenehmen Erscheinungen lässt sich etwas Gutes abgewinnen: Hitzewallungen und Schweißausbrüche in den Wechseljahren scheinen ein Zeichen für ein geringeres Brustkrebsrisiko in der Postmenopause zu sein. In einer Fall-Kontroll-Studie mit 1437 postmenopausalen Frauen, von denen 988 an Mammakarzinom erkrankt waren, waren klimakterische Beschwerden mit einem um 50\% vermindertem Brustkrebsrisiko assoziiert. Das Risiko war umso geringer, je stärker die Wallungen waren. Die Autoren vom Fred Hutchinson Cancer Research Center in Seattle vermuten einen Zusammenhang mit dem Abfall des Östrogenspiegels, der für die Menopausenbeschwerden verantwortlich ist.

Cancer Biomarkers, Epidemiology and Prevention (2011; doi: 10.1158/1055-9965.EPI-10-0998)

www.SpringerMedizin.de

Top gelesen von Hausärzten

Loggen Sie sich ein auf SpringerMedizin.de!

Die jüngsten Lese-Hits:

- Safer Sex muss sein! Lecktuch und Handschuhe sichern lesbische Liebe (325436)

- Weltweites Verbot gefordert: "Coole" Jeans machen Arbeiter schwerkrank (325266)

- Riskante Praxis: Wie geteilte Tabletten Patienten gefährden (325040) Die o.g. Artikel finden Sie, indem Sie den Titel oder die (in Klammern gesetzte) ID-Nummer in die Suche eingeben. 